\title{
Circulation autoantibody against Lamin A/C in patients with Sjögren's syndrome
}

\author{
Wen Zhang ${ }^{1, *}$, Chunyan Zhang ${ }^{2, *}$, Peng Chen ${ }^{1}$, Chunhe Yang ${ }^{1}$, Xianfeng Gan ${ }^{1}$, \\ Muhammad Hussain ${ }^{1}$, Yiping Xun ${ }^{1}$, Yaping Tian² and Hongwu Du ${ }^{1}$ \\ ${ }^{1} 112 \mathrm{Lab}$, School of Chemistry and Biological Engineering, University of Science and Technology Beijing, Beijing, China \\ 2 Department of Clinical Biochemistry, Chinese PLA General Hospital, Beijing, China \\ * These authors have contributed equally to this work \\ Correspondence to: Hongwu Du, email: hwdu93@126.com \\ Yaping Tian, email: tianyp@301hospital.com.cn \\ Keywords: autoimmune diseases; Sjögren's syndrome; Lamin A/C; autoantigen; similar antigen epitopes; Pathology Section \\ Received: April 15, $2016 \quad$ Accepted: October 25, $2016 \quad$ Published: November 09, 2016
}

\section{ABSTRACT}

Lamin A/C proteins are major components of nuclear laminae and were encoded by the LMNA gene. Recent studies have found that in addition to provides nuclearmembrane strength; it also regulates the gene expression. Lamin $A / C$ has been confirmed as an autoantigen in RA, SLE and vasculitis. Anti-Lamin A/C antibodies also have been found by indirect immunofluorescence method. In this study, we used various research methods to confirm Lamin A/C is an autoantigen in Han Chinese patients with confirmed Sjögren's syndrome (SS). To further investigate the relationship between the autoimmune disease antigens, we compared the amino acid sequence of Lamin A/C epitope and several common antigens' antigenic determinant. As a result, we found that Lamin A/C has similar epitopes with U1RNP. It means that the potential relationship exist between Lamin A/C and U1RNP. Clinical data we collected also showed that anti-Lamin A/C and anti-U1RNP antibodies always appear in same serum sample. Therefore, we speculated that cross-reaction may take place between antigen and potential antigen, which have similar epitope. Then, by epitope spreading, the potential antigen can be a new autoantigen. Our study provided a new thinking for further research about the relationship between autoantigens and their development mechanism in autoimmune diseases.

\section{INTRODUCTION}

Sjögren's syndrome (SS) is a systemic autoimmune disease (AID) [1]. Dryness of the eyes and mouth are the main symptoms of SS [2]. Although a lot of important work had been reported in this field, currently the exact mechanism of SS is still unclear [3]. According to AECG [4] and ACR [5] classification criteria, the detection of autoantibodies is an important indicator for $\mathrm{SS}$ clinical classification. Anti-Lamin A/C, as a common autoantibody, associated with a variety of systemic symptoms and considered to play a pathological role in some AID pathogenesis like RA [6], systemic lupus erythematosus (SLE) [7] and vasculitis [8].

It is known that Lamin $\mathrm{A} / \mathrm{C}$ provides nuclearmembrane strength as one of the main proteins of the nuclear envelope. In addition, regulation of gene expression has become its more important function. Lamin $\mathrm{A} / \mathrm{C}$ interacts with histones and DNA in transcription and replication [9]. Also, it is the cause of laminopathies when mutations in LMNA (gene encodes Lamin A or C) take place [10]. Lots of reports hold the same viewpoint that Lamin A/C mutations could alter the differentiation potential of mesenchymal stem cells (MSCs) [11, 12]. MSCs were found from stromal cells of bone marrow by Friedenstein and colleagues for the first time in 1987 [13]. MSCs differentiate into bone, fat and cartilage. MSCs also contribute to maintain the homeostasis of immunological memory by controlling the memory of T cells and plasma cells in a non-proliferative state [14-16]. Clinical trials are in progress, which are expected to use MSCs treating autoimmunity. In a recent report, in 2015, Lamin A/C acts 
as an essential factor in MSCs differentiation through the regulation of the dynamics of the wnt/ $\beta$-catenin pathway and it had been demonstrated [17].

So, it is necessary to find out the significant relationship between Lamin $\mathrm{A} / \mathrm{C}$ and autoimmune diseases. Since Lamin A/C has been confirmed as an autoantigen in RA, SLE and vasculitis, anti-Lamin A/C antibodies also have been found by indirect immunofluorescence in serum of SS in other studies. The important role of Lamin A/C in SS hadn't been discussed yet [18, 19].

In this study, we examined anti-Lamin $\mathrm{A} / \mathrm{C}$ antibodies in serum of SS, and discussed the possible relationship between Lamin $\mathrm{A} / \mathrm{C}$ and other autoantigen of SS. Because, anti-Lamin $\mathrm{A} / \mathrm{C}$ antibodies have been found in previous research work and the sensitivities of the detection was $50 \%$ [20]. As to better illustrate our results we set serum samples of RA patients as positive control and verified our findings by Immunological methods including Western blotting, immunoprecipitation and ELISA, to prove that Lamin $\mathrm{A} / \mathrm{C}$ is an autoantigen of SS in Chinese Han population.

\section{RESULTS}

\section{Lamin $\mathrm{A} / \mathrm{C}$ protein is an autoantigen of SS}

$E$. coli BL21 transfected with the cloning vectors of Lamin $\mathrm{A} / \mathrm{C}$ expressed Lamin $\mathrm{A} / \mathrm{C}$, after that the protein was collected and purification. Electrophoresis was performed with $E$. coli, transfected with the lamin gene, liquid and purified protein of Lamin A/C. Blots in the middle lane and right lane are electrophoresis results of $E$. coli and purified Lamin A/C, respectively. Mass spectrum identification showed that the $70-\mathrm{kDa}$ protein was pure Lamin $\mathrm{A} / \mathrm{C}$ (Figure 1) and it means that all the protein used in this test is pure Lamin $\mathrm{A} / \mathrm{C}$.

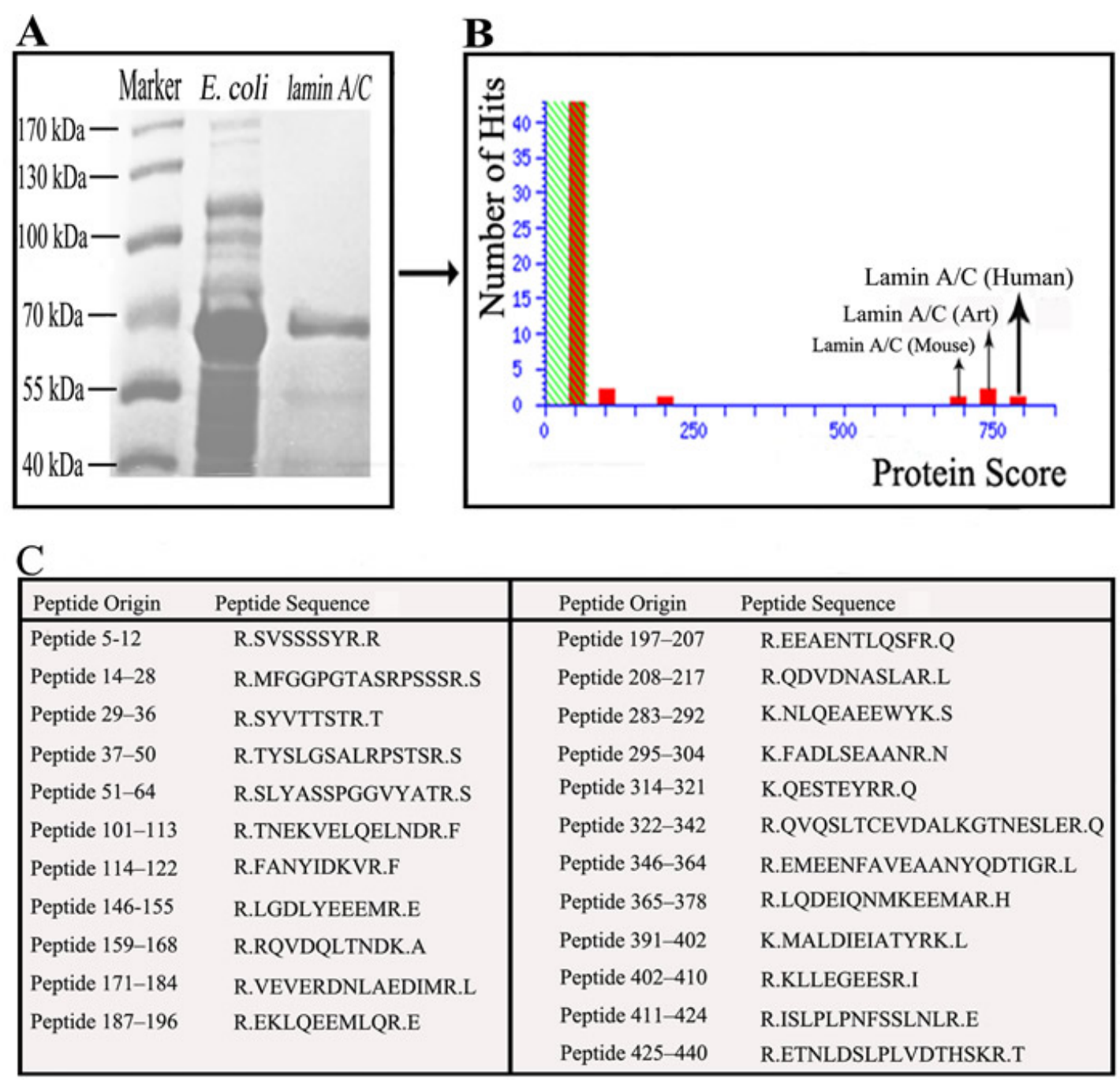

Figure 1: Demonstrate that the 70-kDa protein was pure Lamin A/C. A. Electrophoresed the bacteria and purify the Lamin $\mathrm{A} / \mathrm{C}$, revealed a protein band with about 70-kDa. B. Mass spectrum identification revealed that the 70-kDa protein was pure Lamin A/C. C. 23 Peptides matched Lamin A/C was identified using mass spectrometry. 
5 serum samples from SS patients (Figure 2A) and 5 serum samples from healthy controls (Figure 2B) were performed with Western blotting. In SS serum samples, 2 of $5 \mathrm{IgG}$ autoantibodies to the $70-\mathrm{kDa}$ protein bands were detected, which were absent in healthy controls. In order to further prove that Lamin $\mathrm{A} / \mathrm{C}$ is a potential autoantigen of SS, immunoprecipitation was performed, which showed that a protein band of about $70-\mathrm{kDa}$ could react with SS patient sera (Figure 3). Protein band was excised from the gel, digested with trypsin and identified by using MALDI-TOF-TOF mass spectrometer (Applied Biosystems, Foster City, CA). The data was analyzed with Mascot bioinformatics database search engine (Matrix Sciences, London, UK), proving that the target protein was Lamin A/C.

\section{Prevalence of anti-Lamin A/C antibodies in SS}

Receiver operating characteristic (ROC) analysis between 32 SS patients and 32 healthy controls was carried out to obtain cut-off positivity, whose results indicate that, at value of 0.313 , sensitivity and specificity were 50.00 and 90.62 respectively. Therefore, we set 0.313 as the cut-off value [21]. In the test of ELISA, Lamin A/C was detected in 15 of 24 RA patients $(62.5 \%), 16$ of 32 SS patients $(50.0 \%)$ and 3 of 32 healthy controls $(9.3 \%)$ (Figure 4). Positive rate of SS was higher than healthy controls $(P<0.001)$. In view of the previous study, Lamin $\mathrm{A} / \mathrm{C}$ is a confirmed autoantigen in RA $[22,23]$, so RA was chosen as the positive control. According to results of all tests mentioned above, Lamin $\mathrm{A} / \mathrm{C}$ is an autoantigen of SS.

\section{Amino acid sequence similarities between Lamin $\mathrm{A} / \mathrm{C}$ and other autoantigens}

According to epitope prediction analysis, eight peptides of Lamin $\mathrm{A} / \mathrm{C}$ were predicted as potential epitopes, including 1 to 7 (-METPSQR-), 7 to 21 (-RRATRSGAQASSTPL-), 32 to 37 (-KEDLQE-), 80 to 97 (-AYEAELGDARKTLDSVAK-), 250 to 261 (-AQHEDQVEQYKK-), 382
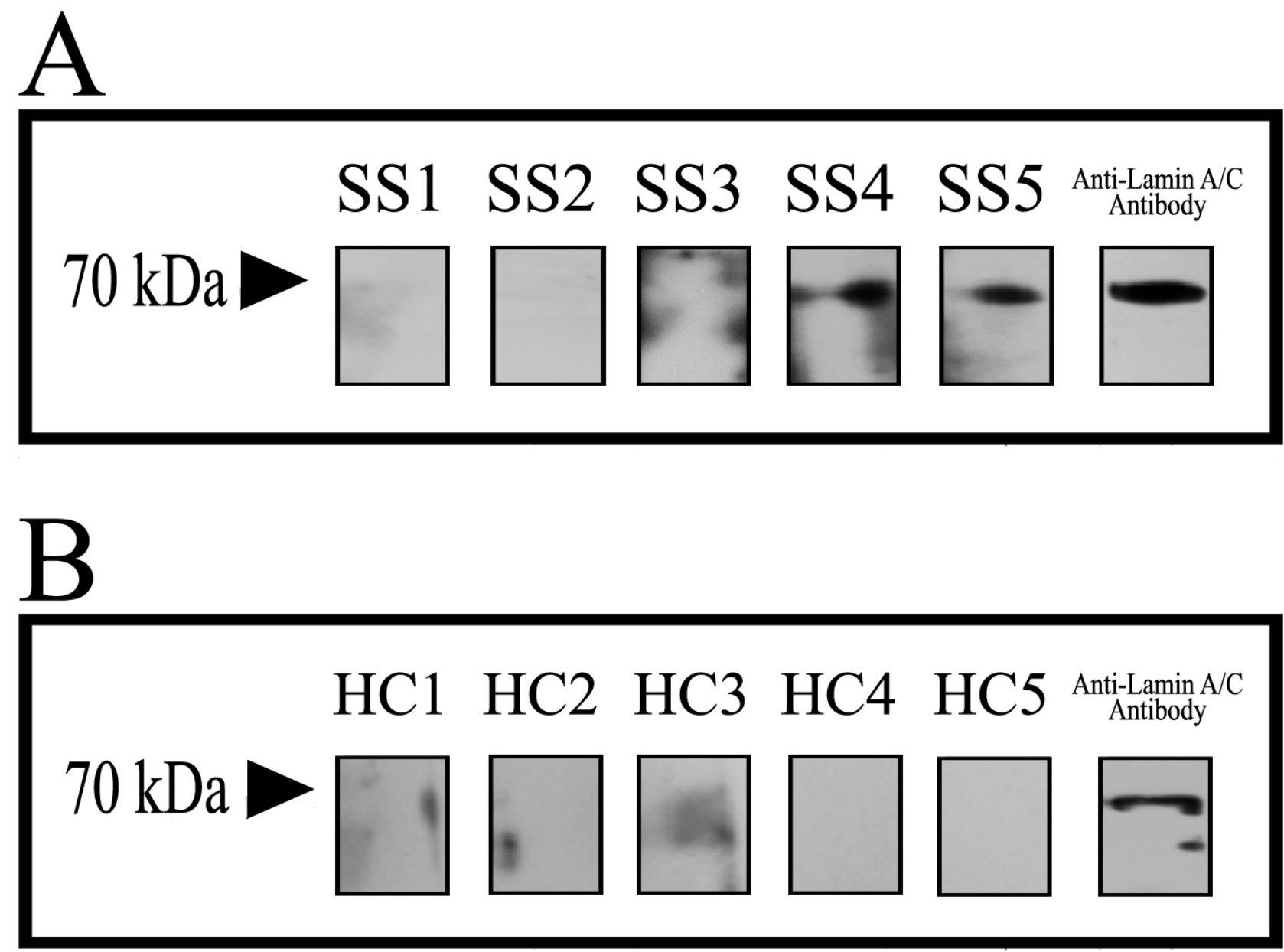

Figure 2: Discover that Lamin A/C is a potential autoantigen of SS. 5 serum samples from SS patients $\mathbf{A}$. and 5 serum samples from healthy controls B. were performed with Western blotting and anti-Lamin A/C antibody in contrast. In SS serum samples 2 of 5 protein bands were detected, but the protein bands were absent in healthy controls. 


\section{Lamin A/C}
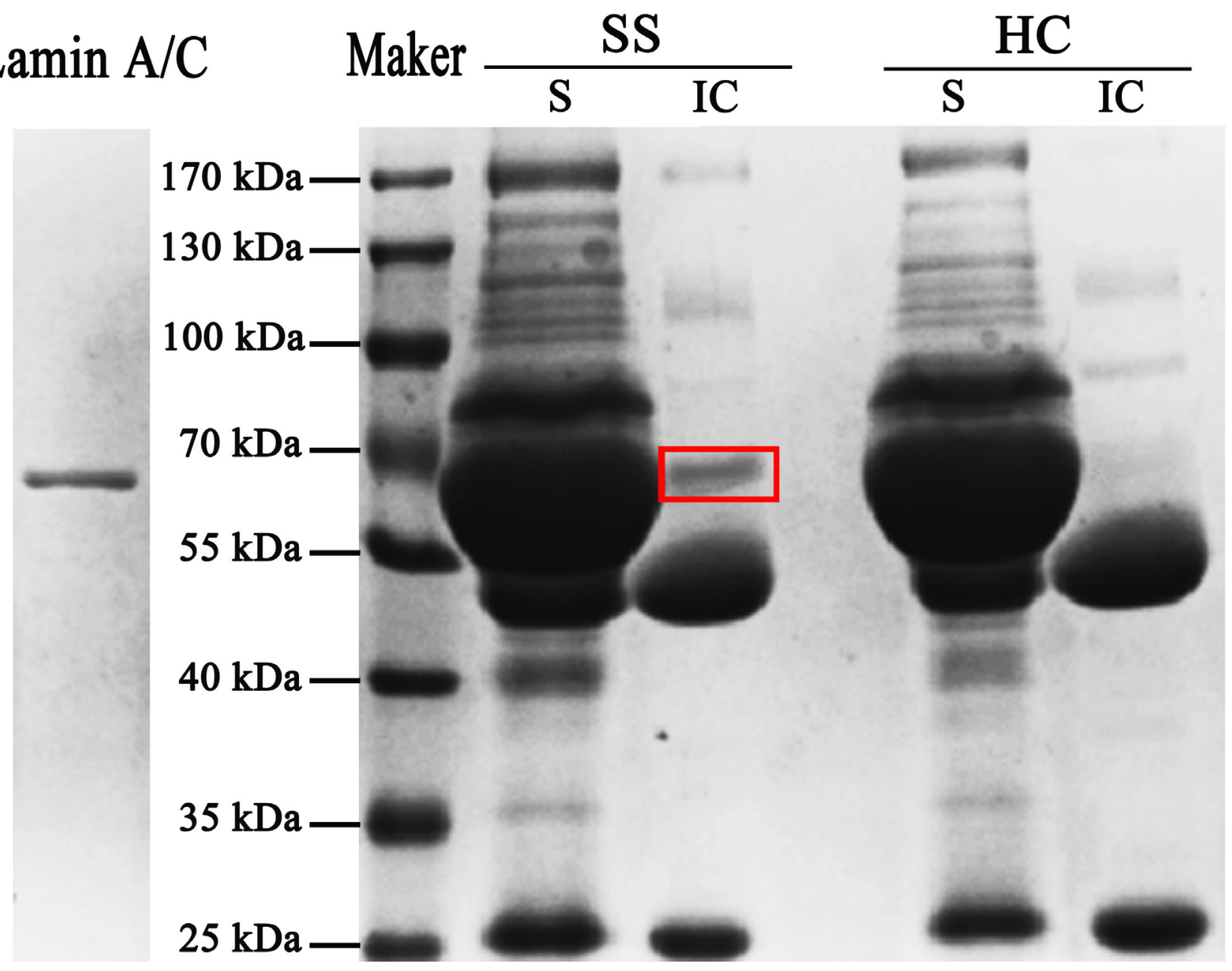

Figure 3: Further prove that Lamin $\mathbf{A} / \mathbf{C}$ is a potential autoantigen of SS. After incubation with the SS and HC sera, the antigen-antibody complexes reacted with protein-A sepharose beads and then electrophoresis was performed with the supernatant (S) and the immune complexes (IC). A protein band of about $70-\mathrm{kDa}$ was present in immune complexes but absent in supernatant.
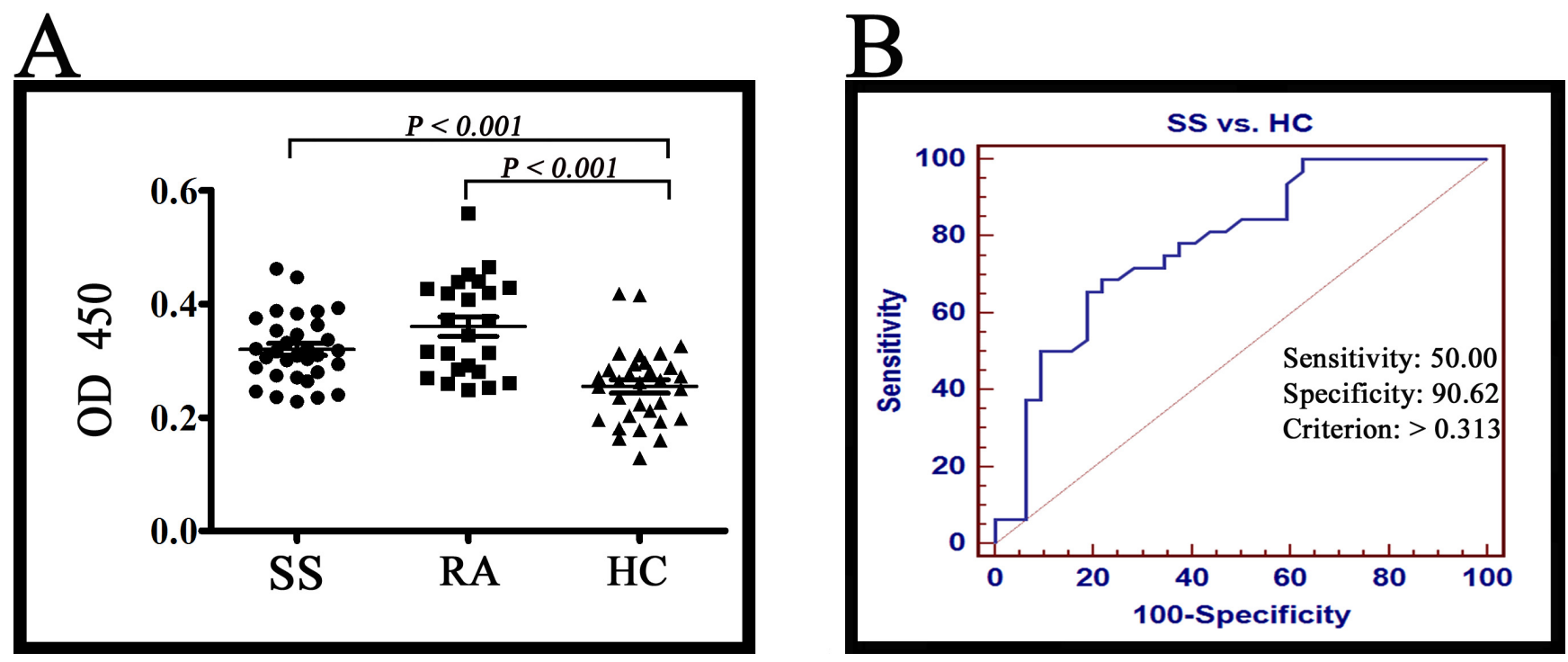

Figure 4: ELISA results. A. The Lamin A/C was detected in 15 of 24 RA patients $(62.5 \%), 16$ of 32 SS patients (50.0 \%) and 3 of 32 healthy controls $(9.3 \%)$. The reactivity of SS serum IgG antibodies against Lamin A/C was higher than healthy controls $(P<0.001)$. B. ROC analysis was carried out for healthy controls and SS patients with MedCalc (MedCalc Software, Mariakerk, Belgium). 
to 394 (-GEEERLRLSPSPT-), 419 to 437 (-RKLESTESRSSFSQHARTS-), 501 to 513 (-GAGATHSPPTDLV-). The results of sequence alignment showed that among eight predicted epitopes some are similar to the confirmed epitopes of SSB, Scl-
70 and U1RNP, respectively (Figure 5). SSA/Ro and Jo-1 also have similar sequence with Lamin A/C, but the confirmed epitope of SSA/Ro and Jo-1 doesn't include in the same sequence.

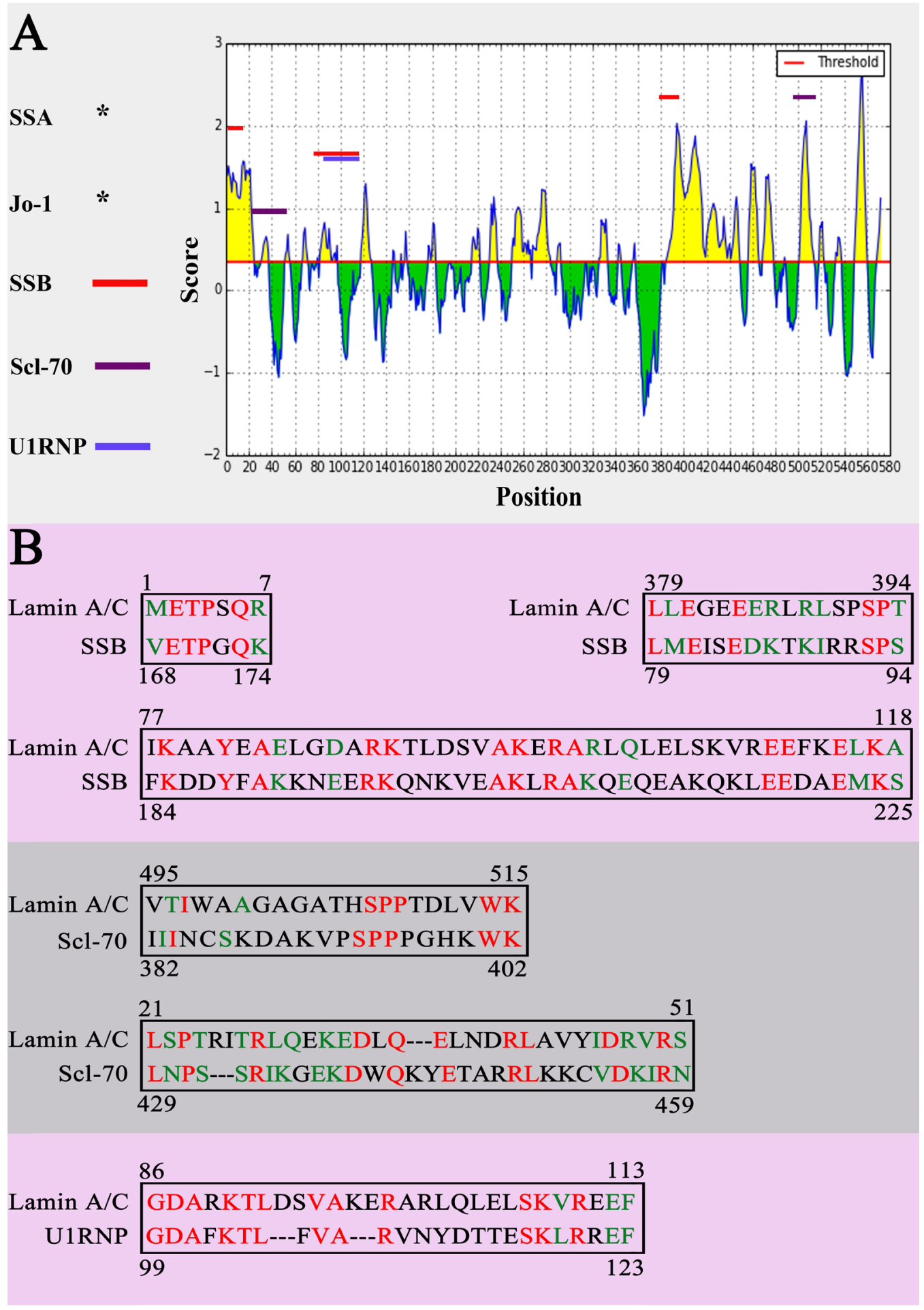

Figure 5: Sequence alignment. A. The sequence alignment results of Lamin A/C with SSB, Scl-70 and U1RNP. B. The same epitopes between potential epitopes of Lamin A/C and antigenic determinants of SSB, Scl-70 and U1RNP. Same amino acid was marked in red. Amino acid with similar properties was marked in green. 
The situation of autoimmune disease associated antibody presented in patients in ELSA test

The heatmap of ELISA and clinical tests showed that the disease results between ELISA, SSA/Ro, SSB/ $\mathrm{La}, \mathrm{Sm}, \mathrm{Jo}-1$ and Scl-70 are discrepant but not significant between ELISA and U1RNP ( $>>0.6)$. Meanwhile the positive rates of the ELISA result $(13 / 24,54 \%)$ and U1RNP (10/24, $42 \%)$ for SS both are apparently higher than other groups (Figure 6). These results demonstrated that anti-Lamin A/C antibody and anti-U1RNP antibody were always present in same serum sample at the same time.

\section{DISCUSSION}

In this study, we have provided further evidences and confirmed that Lamin $\mathrm{A} / \mathrm{C}$ is an autoantigen of SS in Han Chinese patients. Meanwhile some similar antigen epitopes between Lamin A/C and other antigen associated with autoimmune disease were obtained by sequence alignment. Clinical information analysis showed that the

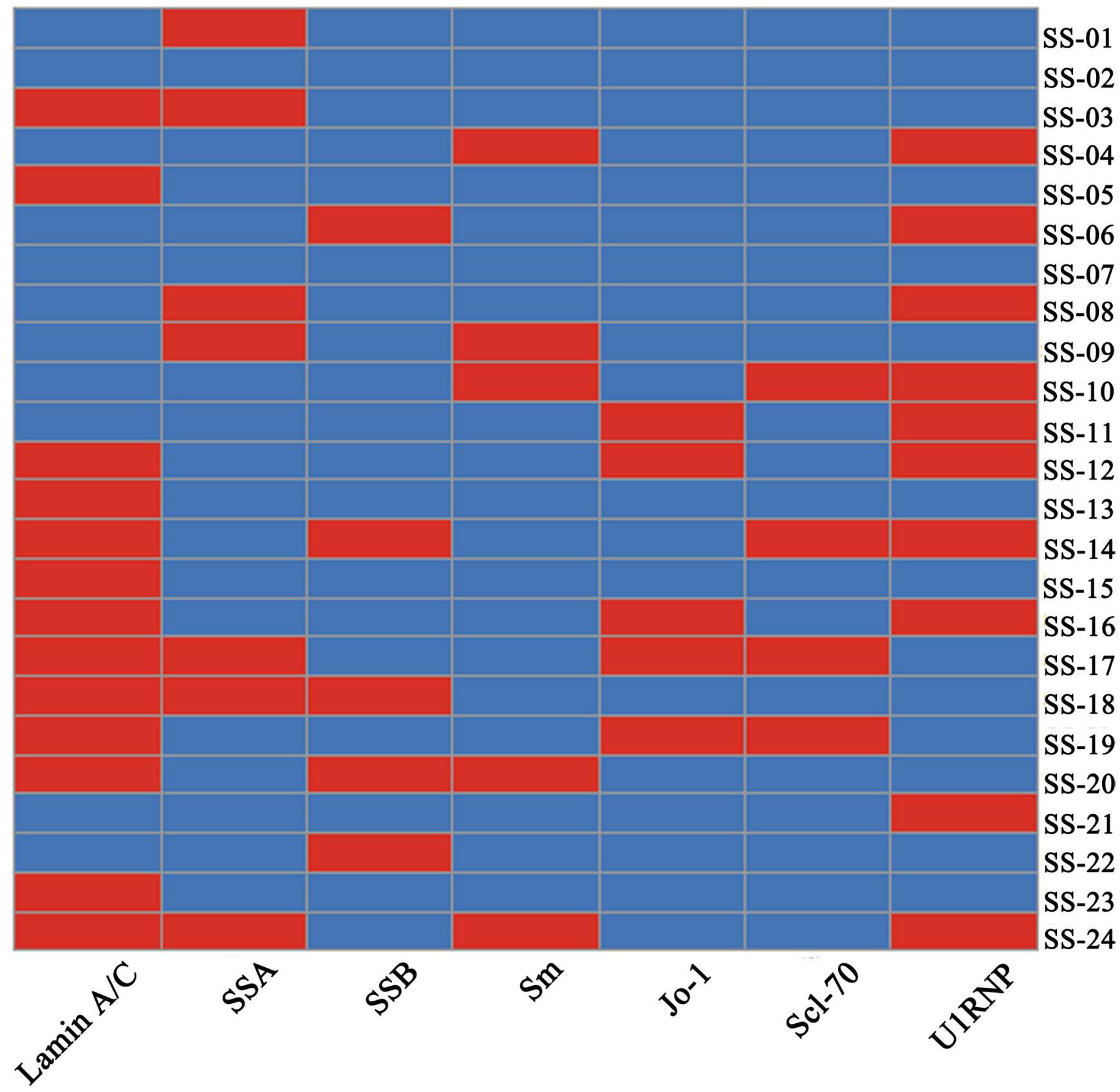

Figure 6: Results of ELISA and clinical test analysis. Five antibody clinical test results of SS patients and ELISA result were analyzed. Every rectangle represents one patient and the red denotes positive but the blue negative. 
presence of anti-Lamin A/C and anti-U1RNP antibody has potential relevancy. This phenomenon may relate the existence of similar antigen epitope between those two proteins.

According to the results of sequence alignment we found that Lamin $\mathrm{A} / \mathrm{C}$ has similar antigen epitope with SSB/La, Scl-70 and U1RNP. Those similar antigen epitopes may play a very important role in autoimmune response. In 2000, Miura and colleagues [24] found two similar epitopes, ${ }^{444}$ TFYLK $^{445},{ }^{458}$ LCENIAGHLK $^{467}$ and ${ }^{445}$ TFYTK $^{449},{ }^{459}$ LCENIANHLK $^{468}$ in bovine and rat catalase, respectively. It also been confirmed that those two similar epitopes are responsible for cross-reaction. That is to say, cross-reaction occur two proteins with similar epitopes. Thus the autoantibody of SSB/La, Scl70 or U1RNP may form immune complex with Lamin A/C protein through recognizing similar antigen epitope.

The theory of epitope spreading holds the view that once the immune tolerance of one protein was abrogated, the autoantibody response could diversify via recognition of new epitopes on this protein [25]. Therefore, when antibodies recognizing similar antigen epitope formed immune complex with Lamin A/C protein, epitope spreading could exposed more epitopes, than Lamin $\mathrm{A} / \mathrm{C}$ became a new target in immune response.

In this study, we also found that the positive rates of ELISA (54\%) and U1RNP (42\%) with 24 patients in clinical information analysis are similar and anti-Lamin $\mathrm{A} / \mathrm{C}$ antibody can be detected in most of the anti-U1RNP antibody positive serum. The similar phenomenon had been observed about half a century ago. Northway [26] and Mattioli [27] found that autoantibodies of RNP and Sm often were found in the same patient sera. It had been proved that both Sm and RNP were located on the U1 snRNP particle [28] and anti-Sm and anti-RNP antibodies were linked [29]. They are always present in the same individual.

Based on the above analysis of the experimental results and clinical information, we inferred that antibodies of a certain antigen could recognize similar epitope on another potential antigen by cross-reaction. Then epitope spreading let more epitope of potential antigen exposed. Finally, this potential antigen becomes a new autoantigen. Of course, the correctness of this assumption needs further researches to confirm. But keeping in view the above facts and findings, this study provides a new thinking of relationship between autoantigens and their development mechanism in autoimmune diseases.

\section{MATERIALS AND METHODS}

\section{Serum samples}

Two categories of serum samples were collected for this study. In a first category 10 serum samples were collected, five from patients of SS with an average age of 70 years (range 59-77) and 5 serum samples from healthy donors with an average age of 27 years (range 24-30). Samples were analyzed by the Western blotting method (Western blotting group). In a second category (validation group) 32 serum samples from patients with SS with an average age of 55 years (range 18-77), 24 serum samples from patients with RA with an average age of 50 years (range 22-77) and 32 serum samples from healthy people with an average age of 26 years (range 21-32) were taken for ELISA test. All the serum samples were collected from the Chinese PLA General Hospital for present study. The ethical committee of Chinese PLA General Hospital has approved this study and consent was taken from each patient. Serum samples were stored at $-80{ }^{\circ} \mathrm{C}$ for further research.

\section{Expression and purification of Lamin $\mathrm{A} / \mathrm{C}$}

The protein expression and purification was performed as described [30].The cloning vector carrying the gene of Lamin A/C was developed as described below.

5'-CAAGAATTCGGTAGCTCCACTCCGCTGT-3' (Upstream primer)

5;-CCGCCGA

GTTATTAATCCTCGTCGTCCTCAAC-3' (Downstream primer)

The cloning vectors were transferred into E.coli BL21 and then the protein of Lamin A/C was expressed efficiently after $6 \mathrm{~h}$ of induction with IPTG. Finally the protein was collected in the insoluble fractions of the cell lysates. The soluble supernatant was passed through NiNTA resin (Qiagen, Hilden, Germany) and BCA assay kit (Biosynthesis Biotechnology, Beijing, China) and was used to determine the concentration of the protein.

\section{Mass spectrum identification}

Mass spectrum was performed as per reference [31]. In this method the band of pure protein was excised from gel followed by destaining with a mixture of $25 \mathrm{mM}$ NH4HCO3 and $50 \%$ acetonitrile. After drying by vacuum centrifugation, the gel pieces were reduced for $2 \mathrm{~h}$ in 10 $\mathrm{mM}$ dithiothreitol. As the temperature cool down, $25 \mathrm{mM}$ NH4HCO3 containing $55 \mathrm{mM}$ iodoacetamide, volume was mixed in the same ratio with the dithiothreitol solution and then incubated for $45 \mathrm{~min}$ at room temperature in the dark. 
Table 1: The clinical information of Sjögren's syndrome patients (SS) were showed as follow, contained the number of patient whose serum presented anti-SSA, anti-SSB, anti-Sm, anti-Jo-1, anti-Scl-70 and anti-U1RNP antibody confirmed by clinical test.

\begin{tabular}{|l|c|c|}
\hline & \multicolumn{2}{|c|}{ SS } \\
\hline Anti-SSA & $7 / 32$ & $21.9 \%$ \\
\hline Anti-SSB & $5 / 32$ & $15.6 \%$ \\
\hline Anti-Sm & $5 / 32$ & $15.6 \%$ \\
\hline Anti-Jo-1 & $5 / 32$ & $15.6 \%$ \\
\hline Anti-Sc1-70 & $4 / 32$ & 12.5 \\
\hline Anti-UIRNP & $10 / 32$ & 31.2 \\
\hline
\end{tabular}

After covering with $20 \mu \mathrm{L}$ of the $0.05 \mathrm{M} \mathrm{NH} 4 \mathrm{HCO} 3$ buffer with trypsin (Sigma, MO), digestion was performed with the gel pieces overnight at $37^{\circ} \mathrm{C}$. The target proteins were identified using MALDI-TOF-TOF mass spectrometer (Applied Biosystems, Foster City, CA), and the data was analyzed with Mascot bioinformatics database search engine (Matrix Sciences, London, UK).

\section{Western blotting}

The purified Lamin $\mathrm{A} / \mathrm{C}$ protein was electrophoresed and then transferred to polyvinylidene fluoride membrane (Merck Millipore, MA) according to standard protocols in our lab [32]. After that the membrane was blocked with $5 \%$ nonfat milk in PBS at $37^{\circ} \mathrm{C}$ for $1 \mathrm{~h}$. The primary antibody (serum samples from 5 SS patients) and 5 healthy controls were diluted with $1 \%$ nonfat milk at a dilution of $1: 1000$, at $4{ }^{\circ} \mathrm{C}$ for overnight. The membrane was extensively washed 3 times with $5 \%$ PBST buffer before reaction with secondary antibody. Anti-human IgG antibody (ImmunoHunt, Beijing, China) as a secondary antibody was diluted with $1 \%$ nonfat milk in a dilution of $1: 10000$ at $37^{\circ} \mathrm{C}$ for $1 \mathrm{~h}$, followed by washing with 5 $\%$ PBST buffer for 3 times again. The Western blotting results were confirmed with enhanced chemiluminescence kit (Applygen, China). The results initially visualized using Gel Doc EZ Imager (Bio-Rad), and then processed using Adobe Photoshop CS3.

\section{Immunoprecipitation}

Immunoprecipitation was performed as per reference [31]. The Lamin A/C (4 mg) was incubated with SS and HC (Healthy Control) sera (equal volumes from $1 \mathrm{SS}$ patients with positive $70-\mathrm{kDa}$ band) at $4{ }^{\circ} \mathrm{C}$ for overnight. After that $20 \mu \mathrm{L}$ protein-A sepharose beads (Sigma, MO) were added at $37^{\circ} \mathrm{C}$. In order to obtain the immune complexes, the solution was centrifuged for $5 \mathrm{~min}$ at $5000 \mathrm{rpm}$. The supernatant was collected from step first and added with $20 \mu \mathrm{L}$ sample loading buffer, then $30 \mu \mathrm{L}$ PBS was added and centrifuged for $5 \mathrm{~min}$ at $5000 \mathrm{rpm}$. Finally, $35 \mu \mathrm{L}$ sample loading buffer was added to the immune complexes. Electrophoresis was performed both, with the supernatant and the immune complexes.

\section{ELISA Test}

The procedure of this method was based on our former work [30, 33]. Lamin $\mathrm{A} / \mathrm{C}$ protein was added into a 96-well microtiter plate (Corning, NY) and incubated with goat sera overnight at $4{ }^{\circ} \mathrm{C}$. The plate was washed three times using PBST. After that, serum samples were diluted 1:100 with the sample buffer and added in the plate for $1 \mathrm{~h}$ at $37^{\circ} \mathrm{C}$. After washing, $100 \mu \mathrm{L}$ of antihuman IgG (ImmunoHunt, Beijing, China) was added, and then incubated for $1 \mathrm{~h}$ at $37^{\circ} \mathrm{C}$. Later, the plate was washed again using PBST, and added with $100 \mu \mathrm{L}$ TMB solution. After incubation for $10 \mathrm{~min}$ at room temperature, $50 \mu \mathrm{L}$ of $2 \mathrm{M} \mathrm{H}_{2} \mathrm{SO}_{4}$ was added. Finally, the absorbance was measured with a plate reader at $450 \mathrm{~nm}$ (Tecan, Hombrechtikon, Switzerland).

\section{Epitope prediction analysis and protein database search}

Confirmed epitopes of SSA/Ro, SSB/La, Sm, Jo-1, Scl-70 and U1RNP were obtained from Immune Epitope Database (IEDB) [34], and antigenic peptides of Lamin $\mathrm{A} / \mathrm{C}$ were also predicted using BepiPred linear epitope prediction at the immune epitope database analysis resource platform [35]. Available sequence alignment tool blast program at NCBI was used to evaluate their homologous sequences. Then these sequences were checked whether they exist or not in confirmed epitopes of SSA/Ro, SSB/La, Sm, Jo-1, Scl-70 and U1RNP, and predicted antigenic peptides of Lamin A/C.

\section{Clinical information analysis}

The clinical informations of patients in ELISA test were collected from the Chinese PLA General Hospital (Table 1). Among 32 patients in ELISA test, there are 8 patients who haven't been tested for the other autoimmune 
diseases related antibody. So, there were 24 samples in clinical information analysis. The results of protein concentration test for SSA/Ro, SSB/La, Sm, Jo-1, Scl70 and U1RNP were confirmed clinically to positive or negative. ELISA test also classify patients into positive or negative. Then the differences of defined results for SS were checked whether the distribution of paired results is significant or not and the heatmap of these results for SSA/ Ro, SSB/La, Sm, Jo-1, Scl-70 and U1RNP were obtained in the $\mathrm{R}$ statistical programming language.

\section{Statistical analysis}

Fisher's exact test was carried out to evaluate the differences between cohorts with Graph Pad Prism (JMP, Cary, NC). McNemar's test was applied to paired test for consistency among different groups with R statistical programming language. It was considered statistically significant when $\mathrm{P}$ values were less than 0.05 . ROC curve analysis was performed between SS patients and healthy controls with MedCalc 9.2 (MedCalc Software, Belgium).

\section{ACKNOWLEDGMENTS}

This work was supported by the National Natural Science Foundation of China (No. 81571592, 31371203 and 81501821), Beijing Nova Program of Science and Technology (No. 2007B024) and Program for New Century Excellent Talents in University (No. NECT-120773).

\section{CONFLICTS OF INTEREST}

The authors declare no financial or commercial conflicts of interest.

\section{REFERENCES}

1. Bombardieri S, Baldini C. Mucocutaneous Manifestations of Sjogren's Syndrome. Handbook of Systemic Autoimmune Diseases, 2006; 5: 147-160.

2. Harley JB, Reichlin M, Arnett FC, Alexander EL, Bias WB, Provost TT. Gene interaction at HLA-DQ enhances autoantibody production in primary Sjogren's syndrome. Science. 1986; 232: 1145-1147.

3. Delaleu N, Jonsson MV, Jonsson R. Disease mechanisms of Sjögren's syndrome. Drug Discovery Today Disease Mechanisms. 2004; 1: 329-336.

4. Vitali C, Bombardieri S, Jonsson R, Moutsopoulos MH, Alexander LE, Carsons SE, Daniels TE, Fox PC, Kassan SS, Pillemer SR, Talal N, Weisman MH. Classification criteria for Sjögren's syndrome: a revised version of the European criteria proposed by the American-European Consensus Group. Annals of the Rheumatic Diseases, 2002;
61: 554-558.

5. Shiboski SC, Shiboski CH, Criswell L, Baer A, Challacombe S, Lanfranchi H, Schiødt M, Umehara H, Vivino F, Zhao Y, Dong Y, Greenspan D, Heidenreich AM, et al. American College of Rheumatology classification criteria for Sjögren's syndrome: A datadriven, expert consensus approach in the Sjögren's International Collaborative Clinical Alliance Cohort. Arthritis Care \& Research, 2012; 64: 475-487.

6. Lassoued S, Oksman F, Fournie B, Danon F, Fournie A, Lassoued K. Autoantibodies to lamins in rheumatoid arthritis. Arthritis \& Rheumatism. 1990; 33: 877-879.

7. Nesher G, Margalit R, Ashkenazi YJ. Anti-nuclear envelope antibodies: Clinical associations.Seminars in Arthritis \& Rheumatism. 2001; 30: 313-320.

8. Régent A, Lofek S, Dib H, Bussone G, Tamas N, Federici C, Broussard C, Guillevin L, Mouthon L. Identification of target antigens of anti-endothelial cell antibodies in patients with anti-neutrophil cytoplasmic antibody-associated vasculitides: a proteomic approach. La Presse Medicale. 2013; 42: 651-652.

9. Dittmer T, Misteli T. The lamin protein family. Genome Biology. 2011; 12: 222.

10. Schreiber KH, Kennedy BK. When lamins go bad: nuclear structure and disease. Cell. 2013; 152: 1365-1375.

11. Malashicheva AB, Zabirnik AS, Smolina NA, Dmitrieva RI, Kostareva AA. Lamin A/C mutations alter differentiation potential of mesenchymal stem cells. Cell \& Tissue Biology. 2013; 7: 325-328.

12. Pekovic V, Hutchison CJ. Adult stem cell maintenance and tissue regeneration in the ageing context: the role for A-type lamins as intrinsic modulators of ageing in adult stem cells and their niches. Journal of Anatomy. 2008; 213: 5-25.

13. Friedenstein AJ, Chailakhyan RK, Gerasimov UV. Bone marrow osteogenic stem cells: in vitro cultivation and transplantation in diffusion chambers. Cell \& Tissue Kinetics. 1987; 20: 263-272.

14. Tokoyoda K, Aenakayama H. Organization of immunological memory by bone marrow stroma. Nature Reviews Immunology. 2010; 10: 193-200.

15. Uccelli A, Moretta L, Pistoia V, Mesenchymal stem cells in health and disease. Nature Reviews Immunology 2008; 8: 726-36.

16. Meirelles LDS, Fontes AM, Covas DT, Caplan AI, Mechanisms involved in the therapeutic properties of mesenchymal stem cells. Cytokine \& Growth Factor Reviews 2009; 20: 419-427.

17. Bermeo S, Vidal C, Zhou H, Duque G. Lamin A/C Acts as an Essential Factor in Mesenchymal Stem Cell Differentiation through the Regulation of the Dynamics of the Wnt/ -Catenin Pathway. Journal of Cellular Biochemistry. 2015; 116.

18. Ou Y, Enarson P, Rattner JB, Barr SG, Fritzler MJ. The nuclear pore complex protein $\mathrm{Tpr}$ is a common autoantigen 
in sera that demonstrate nuclear envelope staining by indirect immunofluorescence. Clinical \& Experimental Immunology. 2004; 136: 379-387.

19. Konstantinov K, Halberg P, Wiik A, Høier-Madsen M, Wantzin P, Ullman S, Galcheva-Gargova Z. Clinical manifestations in patients with autoantibodies specific for nuclear lamin proteins. Clinical Immunology Immunopathology. 1992; 62: 112-118.

20. Gomès-Daudrix V, Sebbag $M$, Girbal E, Vincent $C$, Simon M, Rakotoarivony J, Abbal M, Fournié B, Serre G. Immunoblotting detection of so-called 'antikeratin antibodies': a new assay for the diagnosis of rheumatoid arthritis. Annals of the Rheumatic Diseases. 1994; 53: 735742.

21. Takizawa S, Endo T, Wanjia X, Tanaka S, Takahashi M, Kobayashi T. HSP 10 is a new autoantigen in both autoimmune pancreatitis and fulminant type 1 diabetes. Biochem Biophys Res Commun. 2009; 386: 192-196.

22. Lassoued S, Oksman F, Fournie B, Danon F, Fournie A, Lassoued K. Autoantibodies to lamins in rheumatoid arthritis. Arthritis Rheum. 1990; 33: 877-879.

23. Senecal JL, Raymond Y. Autoantibodies to major and minor nuclear lamins are not restricted to autoimmune diseases. Clinical Immunology \& Immunopathology. 1992; 63: 115-125.

24. Miura H, Tobe T, Miura K, Kobayashi K, Higashi T. Identification of Epitopes for Cross-reaction, Auto-reaction and Autoantibodies to Catalase. Journal of Autoimmunity. 2000; 15: 433-440.

25. Craft J, Fatenejad S. Self antigens and epitope spreading in systemic autoimmunity. Arthritis \& Rheumatology. 1997; 40: 1374-1382.

26. Northway D, Tan EM. Differentiation of antinuclear antibodies giving speckled staining patterns in immunofluorescence. Clinical Immunology \& Immunopathology. 1972; 1: 140-154.

27. Mattioli M, Reichlin M. Physical association of two nuclear antigens and mutual occurrence of their antibodies: the relationship of the SM and RNAprotein (MO) systems in SLE sera. Journal of Immunology. 1973; 110: 1318-1324.

28. Lerner MR, Steitz JA. Antibodies to small nuclear RNAs complexed with proteins are produced by patients with systemic lupus erythematosus. Proceedings of the National Academy of Sciences. 1979; 76: 5495-5499.

29. Pettersson I, Hinterberger M, Mimori T, Gottlieb E, Steitz JA. The structure of mammalian small nuclear ribonucleoproteins. Identification of multiple protein components reactive with anti-(U1)ribonucleoprotein and anti-Sm autoantibodies. Journal of Biological Chemistry. 1984; 259: 5907-5914.

30. Du H, Chen G, Wang S, Wang S, Li S, Li C, Xun Y, He $\mathrm{C}, \mathrm{Wu} \mathrm{M}$. Immunological screening and characterization of highly specific monoclonal antibodies against $20 \mathrm{kDa}$ hGH. Bioanalysis. 2012; 4: 2161-2168.

31. Chen P, Shi L, Jiang Y, Ji Y, Yan H, Sun S, Xun Y, Chen G, Wang X, Chen W, Du H. Identification of heat shock protein 27 as a novel autoantigen of Behcet's disease. Biochemical and biophysical research communications. 2015; 456: 866-871.

32. Xun Y, Chen P, Yan H, Yang W, Shi L, Chen G, Du H. Identification of prohibitin as an antigen in Behcet's disease. Biochemical and biophysical research communications. 2014; 451: 389-393.

33. Du H, Li C, Jin H, Chen G, Xun Y. Generation and evaluation of antibodies against human MGF E-peptide by reverse phase protein microarray and reverse competitive ELISA. Bioanalysis. 2013; 5: 2269-2275.

34. Vita R, Zarebski L, Greenbaum JA, Emami H, Hoof I, Salimi N, Damle R, Sette A, Peters B. The immune epitope database 2.0. Nucleic acids research. 2010; 38: 143-164.

35. Larsen JE, Lund O, Nielsen M. Improved method for predicting linear B-cell epitopes. Immunome Research. 2006; $2: 2$. 